\title{
Place et logique de fonctionnement des clusters : une étude empirique
}

\author{
Samia Haddad, maître assistante \\ Institut des Hautes Études Commerciales - Carthage, Tunisie
}

\section{INTRODUCTION}

\section{La mise en place des clusters est une stratégie innovatrice en matière de développement régional et local.}

Il est de plus en plus admis que la mise en place des clusters est une stratégie innovatrice en matière de développement régional et local. Selon la littérature scientifique dans ce domaine, les clusters (lesquels peuvent prendre la forme de parcs technologiques, de pôles de compétitivité ou de technopoles) permettent de créer les bases d'une synergie réelle entre les sphères de la recherche scientifique, de la formation universitaire et de la production industrielle. En tant que regroupement d'entreprises, de centres de recherche et d'institutions de formation, le cluster ou technopole serait propice à des externalités positives ou économies de réseau. C'est dans cette perspective que cet article examine la logique du fonctionnement des technopoles dans les pays en développement. Il est structuré en trois parties. La première propose un éclairage des fondements théoriques et conceptuels de l'analyse des technopoles et leur contribution au développement. La seconde met en évidence les caractéristiques des clusters dans les pays du Maghreb et analyse le cas de l'Ecopark de «Borj Cédria », en Tunisie. La troisième partie identifie les apports de cette structure de développement et suggère les actions à mettre en place pour améliorer son efficacité.

\section{Les clusters (lesquels peuvent prendre la} forme de parcs technologiques, de pôles de compétitivité ou de technopoles) permettent de créer les bases d'une synergie réelle entre les sphères de la recherche scientifique, de la formation universitaire et de la production industrielle.

\section{LES TECHNOPOLES ET LE DÉVELOPPEMENT. UN CADRE CONCEPTUEL}

Les technopoles constituent le cadre idéal pour valoriser l'innovation et le progrès technologique. Ainsi, elles contribuent au développement et à la croissance économique des pays. Grâce à une infrastructure technique, logistique, administrative et financière de haute qualité, des réseaux spécifiques et des services à valeur ajoutée, ces pôles de compétitivité stimulent et gèrent le transfert des connaissances et des technologies tout en facilitant la création et la croissance d'entreprises innovantes. Ils favorisent de ce fait la génération et la commercialisation d'innovations, la création et le transfert de connaissances et compétences technologiques, la gestion de réseau et la création de synergies au sein de l'industrie $^{1}$. La création et le développement d'activités innovantes à partir des échanges et coopérations entre des entreprises, des centres de recherche et des établissements de formation constituent les propriétés productives des technopoles en tant qu'outil de valorisation des connaissances en milieu de production.

Grâce à une infrastructure technique, logistique, administrative et financière de haute qualité, des réseaux spécifiques et des services à valeur ajoutée, ces pôles de compétitivité stimulent et gèrent le transfert des connaissances et des technologies tout en facilitant la création et la croissance d'entreprises innovantes. 
Par ailleurs, les conditions de réussite d'un cluster technologique peuvent être analysées selon différentes perspectives. En effet, il n'y aurait pas une «recette » universelle pour initier et développer un cluster industriel ${ }^{2}$. De ce fait, il n'existe pas un modèle global de clusters mais plutôt une multitude de modèles faisant partie de la dynamique d'ensemble du système productif local. Aussi, les fonctions principales des clusters consistent à fournir un capital humain et un capital social ainsi qu'un capital financier et des ressources au soutien des jeunes entreprises innovantes qui font partie du cluster ${ }^{3}$. En s'appuyant sur les avancées de la «nouvelle géographie économique », une étude attribue les conditions d'attractivité des parcs scientifiques ou technopoles à trois catégories de facteurs ${ }^{4}$. D'abord, la première catégorie de facteurs a trait aux conditions initiales. À titre d'exemple, la plupart des parcs scientifiques anglo-saxons sont fondés sur l'extension d'une politique locale de valorisation des ressources scientifiques locales. Dans d'autres cas, comme Sophia-Antipolis en
France, la technopole a été créée ex-nihilo, dans un contexte non industriel. Ceci a pu constituer une difficulté pour pérenniser le processus de croissance locale dans le sens où les interrelations localisées ont été plus faibles. La deuxième catégorie renvoie aux complémentarités des relations internes/externes. Le développement économique local est lié à la fois à des ressources notamment scientifiques, technologiques et locales. Il dépend aussi des espaces de marché où ces ressources sont valorisées ainsi que des mécanismes selon lesquels cette valorisation s'effectue. Enfin, le troisième type de facteur influençant l'attractivité d'un territoire technopolitain réside dans la complémentarité et les interrelations entre les ressources locales. En résumé, le cluster s'avère une locomotive de développement économique et serait à l'origine de retombées durables pour les partenaires, les acteurs et pour la région. Sur ce point, la figure 1 illustre le modèle proposé dans le cadre de cet article .

Figure 1 - Le cluster, une locomotive de développement économique

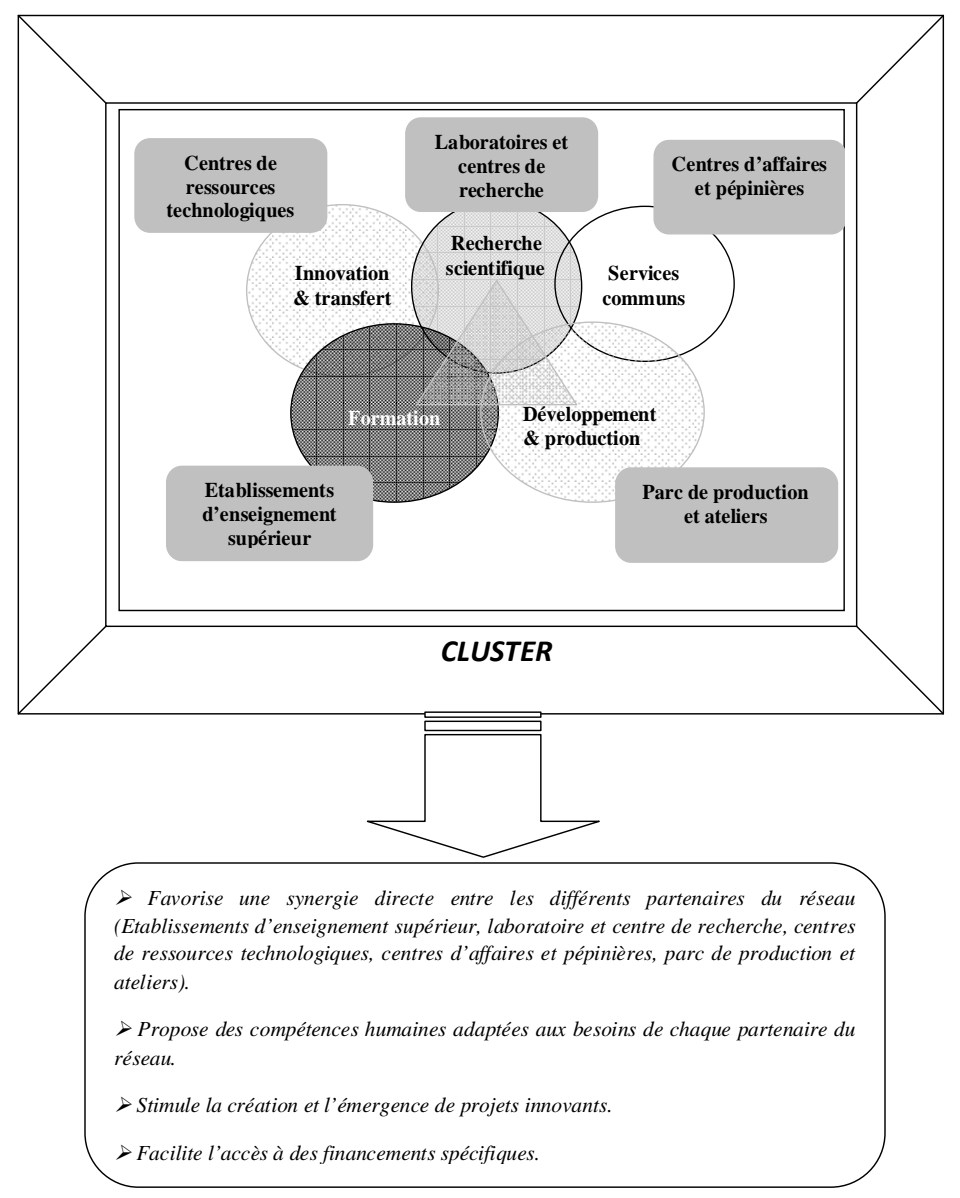


En Tunisie, le développement des technopoles s'inscrit dans le cadre d'une stratégie nationale accordant une priorité absolue à la promotion de l'industrie, de la recherche et de l'innovation dans les secteurs à forte valeur ajoutée.

En Tunisie, le développement des technopoles s'inscrit dans le cadre d'une stratégie nationale accordant une priorité absolue à la promotion de l'industrie, de la recherche et de l'innovation dans les secteurs à forte valeur ajoutée basés sur l'intelligence créative et la haute technologie. La mise en place des technopoles a pour finalité la synergie entre la recherche scientifique, l'enseignement supérieur et le monde des affaires, mais aussi la valorisation des ressources humaines diplômées de l'enseignement supérieur. Dans ce sens, il s'agit de réunir dans un même espace les entreprises privées et publiques, les centres de recherche et les centres de formation, véritables «creuset de cerveaux». Outre les enjeux en matière de développement de la recherche scientifique, la création des technopoles constitue l'édification d'une base économique multidisciplinaire et multisectorielle au niveau local et régional au service du développement.

À partir de la fin des années 90, un réseau de 10 technopoles réparties dans différentes régions du pays a été créé. Chaque pôle répond aux spécificités et aux vocations économiques ainsi qu'aux besoins de développement des régions concernées. Il accorde une place très importante à la coopération internationale et particuliè- rement aux investissements étrangers. Première initiative en matière de parcs technologiques en Tunisie, la technopole «El-Ghazala » a démarré dès 1999 dans la région d'Ariana (une banlieue du Grand Tunis). Elle a accueilli plusieurs activités dans le domaine de la formation, de la recherche scientifique et technologique et dans les domaines de la production et du développement technologiques touchant le secteur des technologies de l'information et de la communication. Ce cluster répondait à un besoin particulier : concrétiser les choix technologiques $\mathrm{du}$ gouvernement tunisien en matière de télécommunications et favoriser l'exportation de l'intelligence tunisienne vers le reste du monde. Suite au succès de l'expérience de la technopole «Elgazala », le gouvernement a décidé de développer, au cours de la période 2004-2009, le concept des pôles technologiques dans les autres régions du pays. C'est dans cette perspective que le processus de création de l'Ecopark «Borj Cédria », spécialisé dans les énergies renouvelables, l'eau, l'environnement et la biotechnologie végétale, doit être perçu. Relevant d'une démarche volontariste, la technopole de «Borj Cédria » s'est appuyée, lors de sa création en 2003, sur des compétences scientifiques.

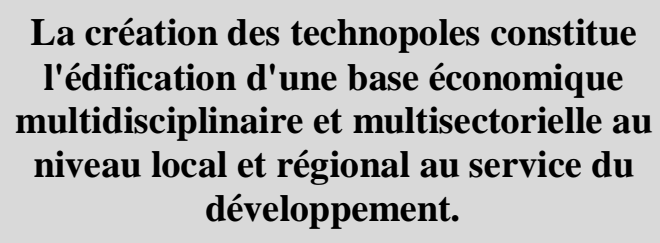

\section{LES CLUSTERS DANS LES PAYS DU MAGHREB : OUTILS DE DÉVELOPPEMENT DE L'INNOVATION TECHNOLOGIQUE?}

En Afrique, le constat autour du développement des clusters est assez pauvre. En 2001, dans le rapport du PNUD, on dénombre seulement 46 pôles, spécialisés dans les TIC, principalement concentrés dans les pays du Nord. L'Afrique n'en compte que deux (Tunisie et Afrique du Sud). Depuis, le mouvement s'est accéléré dans les pays du Maghreb, en particulier en Tunisie qui s'est lancée depuis 1999 dans une stratégie de multiplication des pôles de compétitivités, dans différentes régions, spécialisés dans des domaines high tech (TIC, biotechnologie...). La Tunisie compte actuellement dix technopoles. Le Maroc dispose de sept technopoles (la première, Technopark, créée en 2001). L'Algérie est en train d'aménager une nouvelle ville dédiée aux Nouvelles Technologies d'Informations et de Communications (NTIC).

Par leur positionnement central dans la zone de la Méditerranée, les pays du Maghreb (Algérie, Maroc et Tunisie) permettent l'accès à de 
nombreux marchés. En outre, la proximité géographique de l'Union Européenne reste le facteur déterminant de leur attractivité. Dans ce cadre, les pays du Maghreb se sont mobilisés, depuis le milieu des années quatre-vingt-dix, à renforcer cette attractivité en améliorant la qualité de la main-d'œuvre et des infrastructures, en simplifiant les procédures administratives et en adoptant une législation en faveur des investissements directs étrangers. De tels efforts ont facilité le développement d'un secteur manufacturier traditionnel (textile, habillement, cuir et chaussure) qui s'est progressivement étendu aux industries mécaniques et électriques. Plus récemment, des technopoles sont mises en place dans le secteur des nouvelles technologies (TIC et biotechnologies).

De ce fait, l'évolution des investisseurs étrangers vers de nouveaux secteurs porteurs de valeurs ajoutées, notamment le secteur des biotechnologies végétales, signale la tentative des pays du Maghreb de mobiliser de nouveaux facteurs d'attractivité tels que le capital humain. En effet, au niveau du système éducatif, un important effort a été accompli en vue d'améliorer l'assimilation des progrès technologiques et d'adapter les filières de formation aux besoins des entreprises. Ces efforts portent sur la création des centres de recherche et des centres techniques pour la quasi-totalité des secteurs, la mise en place des technopoles (spécialisées dans les télécommunications, les biotechnologies, l'agro-alimentaire, le textile) et l'accroissement du nombre d'experts de haut niveau.

L'étroitesse du marché a pu être compensée non seulement par la présence d'une forte demande solvable dans les pays de l'Union Européenne, mais aussi par le fort potentiel de développement de nouvelles demandes préférentielles à certains marchés de pays africains (Guinée, Sénégal, Burkina Faso et Niger).

Inéluctablement, les pouvoirs publics dans les pays du Maghreb font des questions du chômage des diplômés de l'enseignement supérieur et du manque d'innovation dans les industries locales des enjeux majeurs de politique publique. La création de technopoles constitue une réponse possible pour faire face à ces nouveaux défis. De ce point de vue, la capacité d'attractivité des technopoles vis-à-vis des entreprises innovantes est stratégique. Ainsi, et dans l'objectif de soutenir et réamorcer une dynamique de croissance, plusieurs États ont entrepris, au début des années 2000, un renforcement des infrastructures de leurs métropoles. Une telle mesure vise à améliorer leur insertion dans l'économie mondiale des flux de connaissances. Ces programmes fédérateurs se situent à la jonction de plusieurs problématiques, telles que la promotion des hautes technologies et de l'innovation, l'aménagement du territoire, le développement durable et l'attractivité vis-à-vis des investisseurs internationaux. C'est dans ce cadre qu'ont émergé les premiers projets de technopoles qui, en associant étroitement entreprises, universités et centres de recherche, font partie intégrante de la stratégie de développement local. Elles sont au cœur de la mise en œuvre d'une «économie de la connaissance » dans la région. Les technopoles ou «parcs technologiques et scientifiques » présentent trois caractéristiques majeures : l'entretien de liens entre les universités et les centres de recherche, l'encouragement au développement d'industries à fort contenu technologique ou de services à forte valeur ajoutée et la promotion du transfert de technologies vers les acteurs locaux. Des programmes de ce type se sont rapidement développés dans le monde tant dans les pays avancés que dans ceux en voie de développement. Les exemples les plus connus étant la Silicon Valley aux États-Unis, Sofia Antipolis en France ou encore le cluster de télécommunication de Rio de Janeiro au Brésil.

L'objectif de la politique des pôles de compétitivité dans les pays du Maghreb est de valoriser les avantages comparatifs régionaux en matière de technologie. Partant d'une réflexion sur le moyen de développer chaque territoire du point de vue technologique, de le rendre plus innovant donc plus attractif, la mise en relation sur une zone géographique de tous ces acteurs semble être une solution adéquate. Il s'avère que chaque pôle de compétitivité est maintenant bâti sur la construction et le développement de projets innovants. C'est le cas notamment de la technopole de «Borj Cédria », fondée en 2003. Sa thématique principale s'articule autour du 
développement durable soit la maîtrise de l'énergie, le traitement de l'eau et les biotechnologies végétales. Il convient alors de décrire la dynamique et logique de fonctionnement de cette technopole.

\subsection{Caractérisation de la technopole de «Borj Cédria »}

La technopole de «Borj Cédria » s'est appuyée sur des compétences scientifiques existantes, une équipe de chercheurs de l'ex-institut national de recherche scientifique et technologique (INRST) qui réalisait de la recherche plutôt fondamentale. À l'origine, elle était axée sur la formation de formateurs et comprenait une centaine de chercheurs. Actuellement, avec l'installation de nouvelles équipes, on en compte environ 400. Ainsi, le parc technologique de "Borj Cédria » comprend quatre espaces : un espace recherche, un espace formation, un espace d'innovation et de valorisation et un espace de production et développement industriel.

\section{L'espace recherche}

L'infrastructure se compose de quatre centres. Le premier est spécialisé dans les technologies de l'énergie avec deux laboratoires et une unité des matériaux. Le deuxième centre, accompagné de quatre laboratoires ${ }^{\mathrm{a}}$ se consacre à l'étude des technologies des eaux. Le troisième centre est axé sur la biotechnologie avec quatre laboratoires et une unité de plantes médicinales et aromatiques. Enfin, le dernier centre en développement se consacre aux sciences des matériaux.

En termes de recherche, la technopole de «Borj Cédria » entretient des relations de coopération avec des partenaires internationaux dans les domaines de la biotechnologie, de l'énergie et de l'eau. L'un de ses objectifs est le réseautage avec des laboratoires étrangers, notamment avec les Japonais. Des experts volontaires japonais et coréens y sont installés ${ }^{\mathrm{b}}$.

Il est important de rappeler la présence, en Tunisie, d'une représentation de la structure de recherche en coopération japonaise, l'Alliance de Recherche en Afrique du Nord de l'université de Tsukuba (ARENA). La coopération avec l'ARENA $^{c}$ s'articule autour de thématiques prioritaires pour la Tunisie, portant notamment sur les biotechnologies végétales. En effet, des projets de recherche en biotechnologie, particulièrement dans le domaine de l'utilisation des plantes pour l'industrie alimentaire, la fabrication de médicaments et de cosmétiques ont été établis entre l'Agence Japonaise de Coopération Internationale (Jica) et la technopole de «Borj Cédria » avec la collaboration de l'université de Tsukuba et l'ARENA. Cette coopération est concrétisée par l'échange d'expériences et d'experts. Sur ce point, Hitoshi Miyazaki, spécialiste en biologie moléculaire à l'université de Tsukuba, met en relief certaines collaborations spécifiques en notant: "nous avons établi des recherches en biotechnologie entre la Tunisie et le Japon depuis deux ans, portant sur les composantes des plantes dans les zones arides en vue de les utiliser dans le domaine industriel et surtout l'élaboration de produits alimentaires, de médicaments..., afin de prévenir ou de traiter certaines maladies comme la cholestérolémie élevée, l'hypertension artérielle, le diabète... »". Actuellement, dans le cadre de l'ARENA, des recherches sur l'olivier sont en cours afin d'élaborer des bio-médicaments, des produits cosmétiques et des produits alimentaires.

\section{L'espace Formation}

Il comprend actuellement l'Institut Supérieur des Sciences et Technologies de l'Environnement (ISSTE) dont l'activité a démarré en 2004. Il représente, aujourd'hui, l'unique institut spécialisé en environnement en Afrique. Eu égard à la complexité des problématiques environnementales, la formation au sein de l'institut est polyvalente et interdisciplinaire. Soucieux de dispenser une formation au diapason des problématiques et stratégies environnementales, l'ISSTE a fait de la coopération avec le milieu socio-professionnel son credo. Ainsi, des professionnels de l'environnement ont été impliqués dans les phases d'élaboration et d'implémentation des programmes d'enseignement. Actuellement, les études à l'ISSTE sont sanctionnées par le Diplôme Universitaire Technologique (DUT) pour répondre aux besoins du marché de l'emploi. Aussi, l'ISSTE est appelé à diversifier ses filières de formation par la création de licences appliquées et de masters professionnels. 
L'espace formation comptera deux autres instituts à savoir l'Institut Supérieur d'Informatique et des Technologies de la Communication (ISITC) et l'Institut Supérieur des Études Technologiques (ISET). Le premier a vocation à devenir une école d'ingénieurs. Certaines universités privées souhaitent s'installer dans la technopole. Une trentaine de doctorants bénéficient de bourses japonaises pour étudier au Japon.

\section{L'espace d'innovation et de valorisation}

Cet espace comprend le Centre des Ressources Technologiques (CRT) et la pépinière d'entreprises «INNOTECH». Le CRT est l'unique centre de ce type en Tunisie. Il offre des laboratoires pour les industriels, les étudiants et les chercheurs. Il dispose d'un hall d'expérimentation, un laboratoire d'analyses physicochimique et d'un centre de documentation (de l'ex-INRST) et un centre de calcul.

\section{La pépinière « INNOTECH » gère} actuellement 17 projets dans les domaines de l'environnement, des ressources naturelles et des biotechnologies végétales. Elle accueille des projets émanant d'ingénieurs ayant des projets de collaboration avec les centres de recherche de la technopole, de chercheurs des autres pôles ou encore de grandes entreprises.

La pépinière «INNOTECH » gère actuellement 17 projets dans les domaines de l'environnement, des ressources naturelles et des biotechnologies végétales. Elle accueille des projets émanant d'ingénieurs ayant des projets de collaboration avec les centres de recherche de la technopole, de chercheurs des autres pôles ou encore de grandes entreprises. Par exemple, FESTO Maghreb, grande entreprise mondiale de pneumatiques, a proposé un projet pour bénéficier des avantages accordés par le parc (infrastructure, hébergement, secrétariat, salle informatique et accès internet). La pépinière offre aussi des conseils aux entrepreneurs et de l'aide à la recherche de financement pour l'accomplissement de leur projet. La sélection des projets est assurée par un comité de pilotage.
Quatre spin-off ont déjà émergé de la pépinière dont deux spécialisées dans les biotechnologies végétales. Le domaine d'activité de la première porte sur l'extraction et la purification d'huiles essentielles, d'oléorésines, d'arômes et de principes actifs. Quant à la seconde, elle est spécialisée dans l'utilisation des technologies de pointe dans le conditionnement et le compostage pour les pépinières.

\section{L'espace industriel et de production}

Il s'étend sur 50 ha et comprend des ateliers relais pour l'expérimentation de la production au stade industriel avant l'installation définitive et une zone de production. Les espaces de production seront attribués sous forme de concessions. Le cahier des charges du parc industriel est maintenant établi. Les critères concernant la sélection des projets sont précis. D'abord, l'activité de l'entreprise doit être en accord avec les principes du développement durable. Ensuite, l'activité doit nécessairement comprendre une part de recherche et développement. Enfin, il ne doit en aucun cas s'agir de délocalisation d'entreprise.

En outre, il n'y a pas de règles concernant l'actionnariat. Les entreprises peuvent être publiques, tunisiennes ou même étrangères. Quant aux services communs, ils comprennent une grande bibliothèque centrale, une université virtuelle, un restaurant et une "guesthouse ». Actuellement, environ le quart de la technopole est construit. Le financement a été assuré par un crédit japonais d'environ 95 millions de \$ US.

\subsection{Positionnement et rôle de la technopole de «Borj Cédria » dans la valorisation des connaissances scientifiques}

Accordant un grand intérêt à la fertilisation croisée entre les sphères de la production industrielle, de la recherche scientifique et technologique et la formation universitaire, l'objectif principal de l'Ecopark « Borj Cédria » consiste à favoriser les interactions et assurer les transferts entre la recherche, l'innovation et le monde productif. Ceci génère un environnement adéquat à l'apprentissage interactif. Ainsi, ce cluster est fondamentalement conçu pour promouvoir et appuyer des activités de hautes 
technologies, notamment dans le domaine des biotechnologies végétales. Il contribue ainsi au développement d'une trajectoire stratégique orientée vers la synergie des réseaux d'acteurs, plus précisément entre les organismes de la recherche et le milieu des affaires.
À la lumière de ce qui précède, l'Écopark étudié est une base multidisciplinaire et multisectorielle de coopérations variées et de valorisation industrielle, tel que schématisé à la figure 2.

\section{Figure 2 - Composantes de l'Écopark « Borj Cédria»}

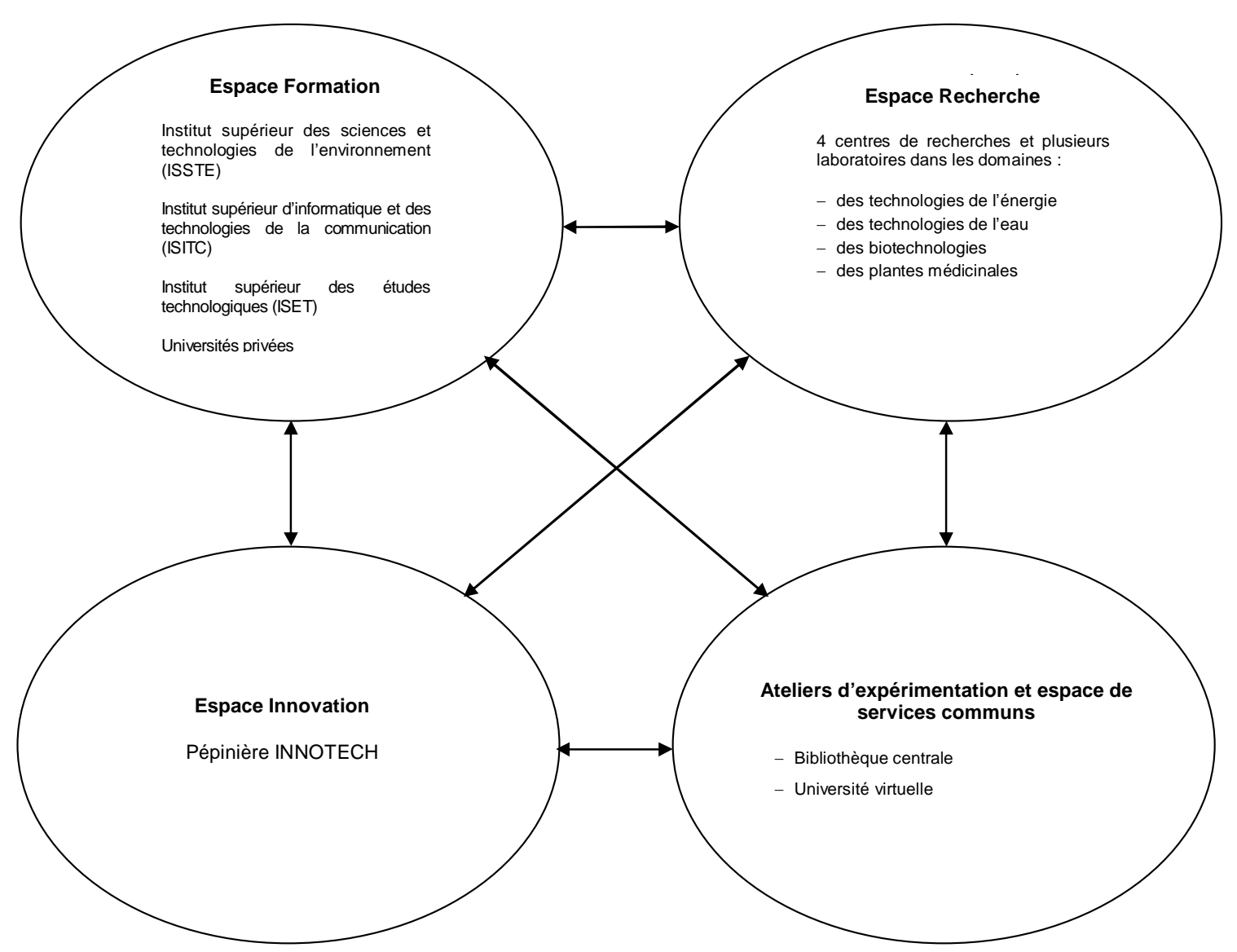

Quelques exemples de valorisation industrielle à partir des connaissances scientifiques méritent d'être mentionnés. Des chercheurs de l'unité des plantes aromatiques et médicinales se sont lancés dans le processus de création d'une spinoff spécialisée dans les biotechnologies végétales. Encore au stade de l'incubation, ce projet industriel est hébergé dans la pépinière «INNOTECH ». Le recours à un partenariat avec des étrangers est en cours de concrétisation. Il permet à ce spin off de bénéficier d'un apport technologique pour parfaire le processus d'extraction des huiles essentielles.
L'autre exemple est celui d'une équipe de chercheurs tunisiens étant parvenue en collaboration avec des Japonais à mettre en évidence un principe actif extrait d'une variété d'huile d'olive tunisienne. Ce principe s'est avéré très performant dans l'inhibition du processus de prolifération de cellules animales atteintes de cancer du sang ou leucémie. Cette découverte, qui couronne les efforts de recherche des équipes tuniso-japonaises, a fait l'objet d'un brevet d'invention international enregistré au Japon. 


\section{LES AVANTAGES DE LA TECHNOPOLE «BORJ CÉDRIA »}

Même s'il est prématuré de procéder à une évaluation détaillée des avantages de la technopole, certaines performances et retombées méritent d'être évoquées. En assumant un rôle dans le développement des marchés, la technopole a permis de développer le marché de l'huile d'olive tunisienne en tant qu'aliment-santé ayant des propriétés anti-oxydantes, notamment sur le marché japonais. Une autre retombée concerne également les briques de Tozeur dont les propriétés particulières (elles ne nécessitent pas de cuisson) sont mises en valeur par leur utilisation dans la construction des bâtiments de la technopole. La légitimation scientifique de propriétés connues empiriquement joue ainsi un rôle marketing non négligeable.

En cultivant, la coopération et l'innovation, plusieurs projets de recherche ont abouti à des dépôts de brevets. Ainsi, les feuilles de certaines variétés d'oliviers tunisiens présentent des propriétés favorables à la réduction de la tension et des cancers du sang (leucémie). Un brevet a été déposé au Japon. Ces propriétés sont actuellement utilisées au titre de la prévention. De nouvelles recherches sont nécessaires pour la mise au point d'un médicament. Les compétences disponibles actuellement en matière de développement de médicaments ne permettent

\section{CONCLUSION}

Cette étude a examiné les fondements théoriques de l'impact des technopoles sur le développement économique. Elle a en effet proposé un cadre conceptuel selon lequel le cluster constitue une infrastructure multifonctionnelle favorable à des externalités positives, mais aussi à une valorisation industrielle des recherches scientifiques. Sur le plan empirique, l'étude a centré l'attention sur l'analyse de la logique et dynamique de fonctionnement $\mathrm{du}$ cluster Écopark «Borj Cédria» en Tunisie. Cette technopole s'appuie sur des compétences scientifiques préexistantes. Elle dispose d'infrastructures de qualité, s'insère dans un réseau international et bénéficie déjà d'un certain nombre d'animations et d'échanges. Enfin, des résultats scientifiques non négligeables ont déjà été produits et semblent en voie pas à la Tunisie de le réaliser seule. Le partenariat apparaît pour l'instant incontournable, mais il devrait contribuer à la construction progressive de compétences tunisiennes dans le domaine du médicament.

Dans le but d'améliorer la performance de la technopole, certaines pistes pourraient être envisagées. Sur le plan du suivi des partenariats avec les pays étrangers, il s'agit de définir les modalités de la valorisation des connaissances élaborées dans le cadre de partenariats transnationaux et brevetées conjointement. Il faudrait également préciser les formes d'appropriation des bénéfices de cette valorisation à la construction des compétences nationales, notamment dans le domaine du médicament. L'exemple des feuilles d'olivier est à cet égard particulièrement intéressant.

Quant à la place de la valorisation industrielle dans la technopole, une attention particulière devrait être apportée aux spins-off hébergés à la Technopole. Il s'agirait ici d'identifier de manière plus détaillée leur rôle dans le processus de valorisation des connaissances scientifiques ainsi que les avantages et les problèmes générés par leur localisation au sein de la technopole lors de leur création puis de leur développement.

d'être transformés en innovations industrielles. La technopole Borj Cédria assume un rôle avant-gardiste en favorisant le développement des marchés de produits à haute valeur scientifique dans des domaines variés. La technopole est aussi à l'origine d'innovations brevetées et de nouvelles compétences tunisiennes dans le domaine du médicament.

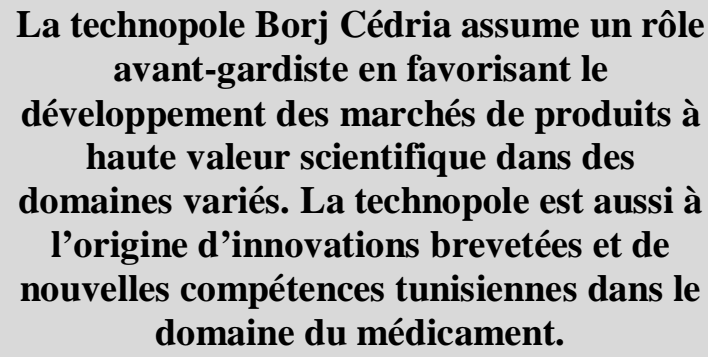
avant-gardiste en favorisant le

développement des marchés de produits à haute valeur scientifique dans des

domaines variés. La technopole est aussi à l'origine d'innovations brevetées et de nouvelles compétences tunisiennes dans le domaine du médicament. 


\section{BIBLIOGRAPHIE}

${ }^{1}$ Hansson, F. (2004). Science Parks as Knowledge Organizations - the ' $b a$ ' in Action? Working Paper, Department of Management, Politics and Philosophy, Copenhagen Business School. Westhead, P., Ucbasaran, D. et Wright, M. (2005). The Focus of Entrepreneurial Research: Contextual and Process Issues. Institute for Enterprise and Innovation, Nottingham University Business School.

${ }^{2}$ Raines, Ph. (2002). Cluster Development and Policy. Aldershot.

${ }^{3}$ Richard, F. (2005). The Flight of the Creative Class: The New Global Competition for Talent. New York: Harper Business.

${ }^{4}$ Queré, M. (2005). «Le développement technopolitain en France. Quels enseignements pour l'attractivité des territoires? ». Revue de l'OFCE, ${ }^{\circ} 94$.

${ }^{5}$ Bel Hadj Ammar, S. et Amami, M. (2007). «Développement de Parcs Technologiques : Enjeux et Déterminants Clés de Performance. Cas Pôle «Elgazala » des Technologies de la Communication », www.attr.org.tn/fr/regardcol/Amma.pdf.

${ }^{5}$ Haddad, S. et Poncet, Ch. (2008). «Clusters et dynamique de l'innovation dans les télécommunications sur le site d'Elgazala en Tunisie », Laperche, B., (Ed.), L'innovation pour le développement. Enjeux globaux et opportunités locales. Edition Karthala, Paris, pp. 93-115.

\section{Notes}

${ }^{\mathrm{a} C e s} 4$ laboratoires sont les suivants : Caractérisation et qualité de l'huile d'olive; physiologie moléculaire de la vigne; adaptation des plantes aux stress abiotiques; interactions légumineuses-microorganismes et une unité de plantes médicinales et aromatiques.

${ }^{\mathrm{b}}$ Cette coopération s'est traduite notamment par l'organisation, en 2006, des journées Tuniso-japonaises de la recherche à laquelle ont assisté plus 350 participants dont une centaine de scientifiques japonais.

'L'ARENA ou l'Alliance de recherche en Afrique du Nord de l'université de Tsukuba est un centre de recherche à l'université de Tsukuba fondé en 2003. Il est composé de 4 sections : la biotechnologie, l'environnement dans les zones arides, l'information et la technologie des communications et, enfin, les arts et la culture. L'ARENA a pour rôle d'établir des échanges scientifiques et académiques entre les institutions supérieures japonaises et les pays africains dont la Tunisie, l'Egypte, la Libye, le Maroc, l'Algérie, la Mauritanie... Un accord-cadre de coopération scientifique et technique entre l'ARENA e1t le Ministère de la Recherche scientifique, de la Technologie et du Développement des Compétences a été signé le 6 juin 2005 à Tunis.

${ }^{\mathrm{d}}$ Propos recueillis à partir de l'article paru dans La Presse du 25 janvier 2007 sur « la recherche dans le domaine de la biologie moléculaire ». 


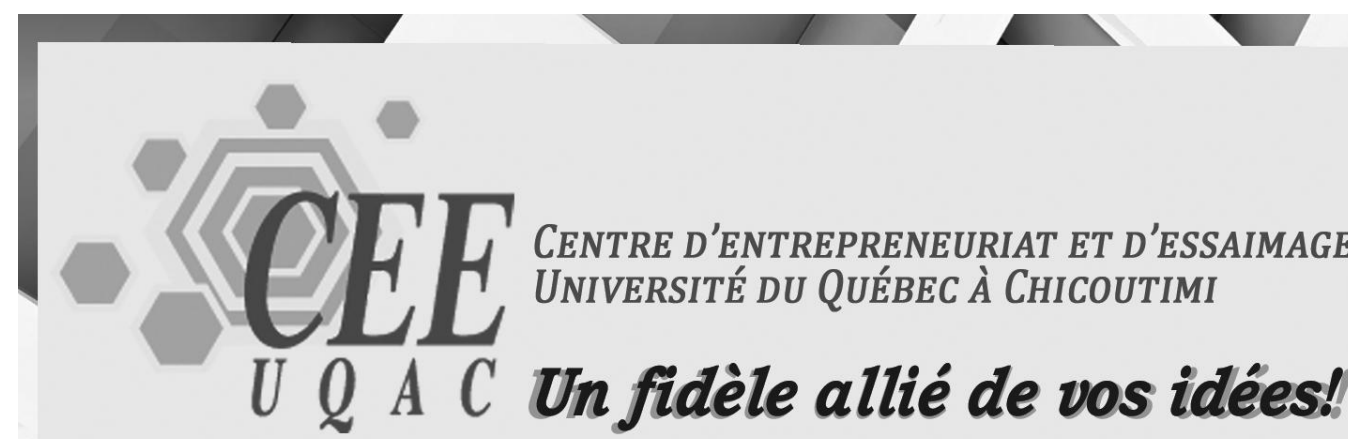

Le CEE-UQAC vous offre :

- Un accompagnement personnalisé en création, démarrage et relève d'entreprise!

- Validation d'idées d'affaires

- Soutien à la réalisation du plan d'affaires

- Des activités à saveur entrepreneuriale!

- Midi-conférences, colloques, salons

- Ateliers d'information et de perfectionnement

- Chroniques entrepreneuriales

- Concours entrepreneuriaux

- Des projets technologiques rémunérés, directement liés à votre champ d'études, qui vous offrent la possibilité de travailler dans les PME régionales!

- Un Réseau international d'affaires à l'UQAC (RIA-UQAC) qui permet aux PME de développer des projets d'affaires avec l'étranger grâce à la collaboration des étudiants internationaux diplômés de l'UQAC!

- Un Centre de développement technologique en jeux vidéo et en informatique (CDT-UQAC) qui contribue à l'émergence de projets innovateurs et au développement d'entreprises en lien avec la conception de jeux vidéo et l'informatique, et ce, par un encadrement d'affaires adapté.

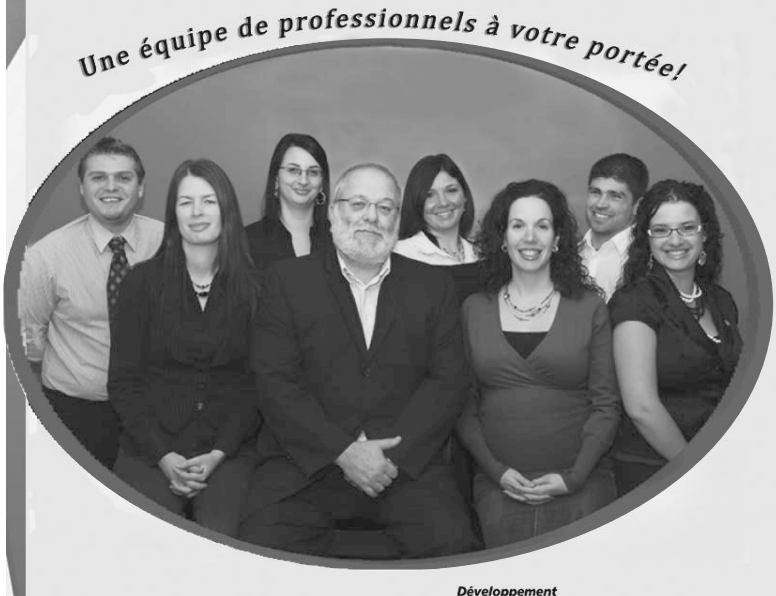

Venez nous rencontrer! CEE-UQAC Chicoutimi

555 , boul. de l'Université, local H1-1180

Pavillon des humanités, UQAC

Chicoutimi (Québec) G7H 2B1

Téléphone : $418545-5011$ poste 4655

Courriel : cee-uqa@@uqac.ca

CEE-UQAC Côte-Nord

Cégep de Sept-Îles, 175, de la Vérendrye

Sept-Îles (Québec) G4R 5B7

Téléphone : 418 968-8387•cee_cn@uqac.ca

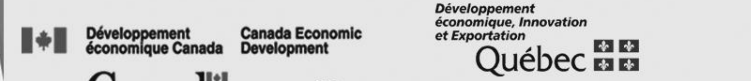

\section{UQAC}

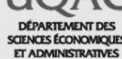

Canadă

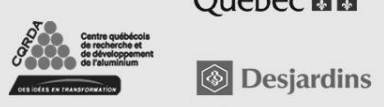

FONDATION

UQAC
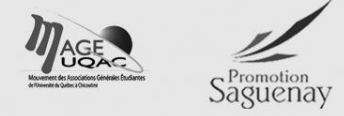